\title{
Trivium
}

Revue franco-allemande de sciences humaines et sociales - Deutsch-französische Zeitschrift für Geistesund Sozialwissenschaften

15 | 2013

La science pense en plusieurs langues

\section{La langue maternelle, moteur de la créativité de la pensée}

\section{Walter Krämer}

Traducteur : Claudine Layre et Anne-Emmanuelle Fournier

\section{(2) OpenEdition}

Journals

Édition électronique

URL : http://journals.openedition.org/trivium/4682

ISSN : 1963-1820

\section{Éditeur}

Les éditions de la Maison des sciences de l'Homme

\section{Référence électronique}

Walter Krämer, «La langue maternelle, moteur de la créativité de la pensée », Trivium [En ligne], 15 |

2013, mis en ligne le 09 décembre 2013, consulté le 07 septembre 2020. URL : http:// journals.openedition.org/trivium/4682

Ce document a été généré automatiquement le 7 septembre 2020.

\section{(c) (1) $\odot$}

Les contenus des la revue Trivium sont mis à disposition selon les termes de la Licence Creative Commons Attribution - Pas d'Utilisation Commerciale - Pas de Modification 4.0 International. 


\title{
La langue maternelle, moteur de la créativité de la pensée
}

\author{
Walter Krämer \\ Traduction : Claudine Layre et Anne-Emmanuelle Fournier
}

1 Notre propos porte sur la recherche et l'enseignement ainsi que sur l'influence réciproque entre ces activités et la langue dans laquelle elles s'effectuent. L'existence de cette influence réciproque ne fait aucun doute. Je me limiterai ici à examiner une seule des deux directions de cette relation, l'influence de la langue sur la pensée, la recherche, l'enseignement, et en particulier le désastre qu'entraîne la monoculture mondiale de l'anglais dans les universités de tous les pays de cette planète où l'anglais n'est pas la langue maternelle.

2 Commençons par les petites catastrophes. Elles se produisent toutes les semaines et tous les jours en d'innombrables établissements d'enseignement, supérieur ou non, quand des chargés de cours maîtrisant insuffisamment l'anglais s'adressent à des étudiants qui sont dans le même cas. Pas plus tard que ce lundi, j'ai assisté à une conférence dans mon université d'origine à Dortmund, où l'orateur invité, un éminent spécialiste allemand, a été prié de s'exprimer en anglais. C'est ce qu'il a fait, tant bien que mal. Aucun débat n'a pu être engagé, j'aurais pu m'épargner ces deux heures.

Mais ce ne sont que de petites catastrophes. Les grandes se produisent en amont. Car avant de présenter le résultat de ses recherches, il faut d'abord en avoir. Et c'est précisément-là, à la naissance de l'idée, lors de la recherche proprement dite, que le renoncement à sa langue maternelle, ou pire l'amalgame de la langue maternelle à l'anglais omniprésent, qui aboutit à un pidgin moderne, provoque les plus grands dégâts.

4 Reconnaissons-le, en croyant que la recherche de pointe ne peut ou ne doit plus être effectuée qu'en anglais, beaucoup de mes homologues allemands contribuent au fait que la recherche de haut niveau ne voit même plus le jour en Allemagne. Ce n'est pas ici l'allemand en tant que langue scientifique internationale qui est en jeu, mais son rôle en tant que langue nationale, en tant que médium dans lequel les chercheurs allemands pensent, ruminent, développent des idées, formulent des hypothèses, établissent des 
transversalités, ont des éclairs intellectuels; il s'agit de l'outil, de l'accoucheur, qui seul permet aux théories et aux idées de quitter le chaos de nos cellules nerveuses pour parvenir au monde extérieur.

5 Je recommande à tous mes homologues qui sont obligés de bafouiller en anglais lors de conférences internationales - et sont de ce fait toujours moins bons que les locuteurs natifs anglophones - de lire l'essai intemporel de Heinrich von Kleist intitulé De l'élaboration progressive des idées par la parole. ${ }^{1}$ Il commence ainsi : "Lorsque tu veux savoir quelque chose et que tu ne peux y parvenir par la méditation, alors je te conseille, mon cher et savant ami, d'en parler avec la première personne qui se présente à toi». ${ }^{2}$ Car selon Kleist, lorsque nous parlons, nos cellules nerveuses sont pour ainsi dire attisées, galvanisées et poussées au sommet de leurs performances; la parole ouvre la porte à la pensée. "Le Français dit: "l'appétit vient en mangeant", et cette maxime fondée sur l'expérience reste vraie quand on la parodie en disant "l'idée vient en parlant" ${ }^{\prime \prime} »$

6 Kleist raconte ensuite une expérience qu'il a vécue plusieurs fois: quand il fait démarrer le moteur du langage, tout simplement en commençant à parler, la langue le porte ensuite d'elle-même, sans qu'il ait besoin de réfléchir, jusqu'à son objectif : « Mais parce que j'ai malgré tout une obscure représentation du problème, liée de plus ou moins loin à ce que je cherche, mon esprit, quand je prends avec fougue la parole en premier et tandis que la conversation progresse, dans la nécessité de trouver une fin au commencement, éclaircit cette représentation nébuleuse en une idée d'une clarté absolue, de sorte que le raisonnement aboutit, à mon grand étonnement, au moment où ma longue phrase s'achève ${ }^{4}$.»

7 Au grand étonnement de Kleist, le raisonnement s'achève avec sa phrase ! Car la langue est plus qu'une interface grâce à laquelle notre pensée entre en contact avec le monde extérieur, la langue est l'un des moteurs mêmes de la pensée, la langue est un facteur de production, et l'utilisation optimale de ce facteur n'est possible pour la plupart des gens que dans leur langue maternelle.

8 Je cite ici mon collègue Ehlich, le rapporteur de cette section $^{5}$ : «La possibilité de mettre en mots est partie prenante du processus cognitif lui-même, et la langue occupe dans l'élaboration de la connaissance une fonction gnoséologique (qui lui est) propre. »

Une langue maternelle malléable, souple, adaptable et innovante est donc indispensable à toute créativité de la recherche. Ce n'est pas un hasard si l'explosion des découvertes scientifiques à la fin de la Renaissance a coïncidé avec le déclin du latin comme langue de réflexion des nations européennes. Galilée pensait en italien, Kepler ou Leibniz en allemand et Newton sans doute en anglais. Seuls les résultats de leur réflexion ont été publiés par eux tous en latin.

$\mathrm{Si}$, non content de confier la transmission du savoir à un pidgin comme celui qui paraît aujourd'hui suffisant à de nombreux scientifiques allemands pour penser notre monde, on lui abandonne aussi le surgissement des idées, alors la recherche de haut niveau n'est plus possible. Je cite ici Josef Weizenbaum du MIT, l'expert en informatique sans doute le plus connu au monde, qui considère lui aussi la façon dont s'expriment les informaticiens allemands comme l'une des raisons du retard de l'informatique allemande par rapport à la recherche américaine dans ce domaine: "Toute personne pense dans sa propre langue, avec les nuances qui lui sont propres. Le fait que de nombreux Allemands soient accros à des bribes d'anglais entraîne au contraire une 
pauvreté linguistique, un parler qui devient une véritable soupe. Dans ces conditions, il n'est pas possible que naissent des idées. "

11 Le point crucial est là : "Dans ces conditions, il n'est pas possible que naissent des idées.» On peut donc renverser le principal argument des défenseurs de l'anglais comme unique langue scientifique (y compris dans les pays situés en dehors de l'espace linguistique anglo-saxon) selon lequel la science allemande doit commencer par progresser afin qu'ensuite la langue allemande se porte mieux. En réalité, c'est tout à fait l'inverse : il faut d'abord que la langue allemande s'améliore; il faut d'abord que nous nous entraînions de nouveau à penser de façon créative et innovante, et à ce moment-là, la qualité de la science allemande s'accroîtra. Car, soulignons-le encore une fois, la plupart des gens ne peuvent penser de manière créative que dans leur langue maternelle, et si celle-ci exclut de son champ des pans entiers de la vie et du savoir, il n'est alors plus possible de penser le monde dans cette langue maternelle.

Aussi, l'argument souvent entendu selon lequel les langues de spécialité seraient incompréhensibles pour les non-spécialistes y compris dans leur langue maternelle, si bien qu'il ne serait pas du tout aberrant de pratiquer directement la science dans une langue étrangère, passe par conséquent à côté du vrai problème. La science a évidemment un devoir d'explication vis-à-vis de ceux qui financent cette entreprise (les électeurs et les contribuables). Or il est plus facile, même si ce n'est pas toujours simple, de s'acquitter de cette dette dans une langue comprise par les financeurs, c'est-à-dire dans leur langue maternelle. Certes, il est regrettable que même dans ce cas-là, il subsiste en général encore des incompréhensions (même si celles-ci sont plus minimes que si tout avait été communiqué d'emblée dans une langue étrangère). Mais cela n'a rien à voir avec le fonctionnement de notre machine à penser qu'est le cerveau. L'enjeu fondamental n'est pas seulement, et pas même en premier lieu, qu'un large public comprenne une idée, mais bien que le producteur de l'idée lui-même la comprenne.

13 Une fois qu'il ou elle l'a comprise, rien n'empêche que celle-ci soit communiquée à un public international dans une langue véhiculaire choisie comme bon lui semble, ce que nos amis français pratiquent depuis longtemps avec succès, en tout cas dans les matières dont je suis moi-même l'actualité. Car en France, la recherche économique et économétrique a lieu en français. La terminologie est vaste et riche, elle favorise la créativité de la pensée et permet à nos homologues français d'élaborer d'abord en français tout ou presque de ce qu'ils peuvent ou veulent penser. Ensuite seulement, le résultat est retranscrit dans l'anglais qu'on leur connaît et qui ne va pas sans accrocs, afin d'être communiqué à un public international. Et ce avec un grand succès. L' Econometric Society, la société internationale de spécialistes la plus renommée dans mon domaine de recherche, compte 78 membres en France dont 28 "fellows». Les fellows sont des membres qui ont été distingués pour leurs remarquables performances scientifiques, et admis dans ce noble cercle des plus grands économistes mondiaux par cooptation. En Allemagne, cette société comprend 166 membres mais seulement 9 fellows. Cela signifie pour moi que si les Allemands sont plus zélés, ils produisent moins de recherche de haut niveau, que leur addiction aux bribes d'anglais dont parle Weizenbaum est un obstacle à l'innovation scientifique. Et dans les faits, quand on écoute le charabia souvent indigeste dans lequel certains chercheurs croient devoir s'exprimer, par exemple lors du congrès annuel de l'Association des sciences économiques et sociales (Verein für Socialpolitik), on a du mal à imaginer qu'il recèle de notables contributions à la recherche. 
Qu'en conclure, sinon que la pénétration de l'anglais dans le milieu scientifique allemand n'est pas une aide mais un frein au progrès de la connaissance ? En suivant cette voie, nous entérinons le déclassement de la recherche allemande dans tous les domaines et nous nous transformons à jamais en subalternes voire en esclaves d'un système de valeurs et de communication international dominé par les AngloAméricains, nous nous muons en citoyens de seconde zone dans notre propre milieu scientifique.

\section{NOTES}

1. Kleist, H. v. (2010) : De l'élaboration progressive des idées par la parole, trad. par B. Germain, Paris : Ed. Sillage.

2. Kleist (2010), p. 25.

3. Kleist (2010), p. 26.

4. Kleist (2010), p. 27.

5. Il s'agit de la section «Lehren und Veröffentlichen in der Landessprache: notwendige Grundlage oder Relikt? / Enseigner et publier dans la langue nationale : fondement nécessaire ou résidu? » de l'ouvrage Europa denkt mehrsprachig/L'Europe pense en plusieurs langues, p. 49-90 [NDLR].

\section{INDEX}

Mots-clés : langue maternelle, recherche créatrice, « De l'élaboration progressive des idées par la parole », monoculture de l'anglais, science allemande

Schlüsselwörter : Muttersprache, kreative Forschung, ,allmähliche Verfertigung der Gedanken beim Reden“, englischsprachige Monokultur, deutsche Wissenschaft

\section{AUTEURS}

\section{WALTER KRÄMER}

Walter Krämer est professeur d'économie et de statistique à l'Université technique de Dortmund. Pour en savoir plus, consulter la notice 